\title{
Computer designed orthotic device for offloading plantar ulcers-Technical paper
}

\author{
M. David Prakash Kumara ${ }^{\mathrm{a}}$, M. Sathish Paul ${ }^{\mathrm{a}} \&$ B. Siva ${ }^{\mathrm{a}}$ \\ ${ }^{a}$ Schieffelin Institute of Health Research \& Leprosy Centre, Karigiri, \\ Vellore, Tamil Nadu, India
}

Submitted 27 January 2021; Accepted 22 April 2021

\begin{abstract}
Summary
Introduction: Plantar ulcers occur in patients with peripheral arterial disease or peripheral neuropathy. Plantar ulcers resulting from the peak plantar pressures often lead to disabilities and even amputations. Using an appropriate offloading device would reduce peak plantar pressures and prevent ulcers.

Objectives: To design and analyze a customized lightweight offloading device that will improve patient compliance and provide good aesthetic value, without compromising on efficacy. Computer Aided Design (CAD) software SOLIDWORKS was used to design two different 3-dimensional models of offloading devices. The light weight, energy efficient and cosmetically appealing offloading devices could be a replacement for the traditional offloading devices made of Plaster of Paris, which are bulky and unappealing, leading to poor compliance.

Results: The computer simulation studies have established the efficacy of the new designs in offloading the foot.

Conclusion: The developed designs could be an ideal replacement for traditional offloading devices like the Bohler iron. The designs could improve the user's compliance and acceptance without compromising on the efficacy. These designs need to be field tested.
\end{abstract}

Keywords: Diabetes, leprosy, neuropathy, offloading, peak plantar pressure, pressure ulcers

\section{Background}

Leprosy is a chronic infectious disease that causes physical deformity resulting in stigmatization of affected individuals. More than 200,000 new cases have been reported each year. ${ }^{1}$ Neuropathy is common in those affected by leprosy and diabetes. Increased mechanical stress concentrated on the plantar tissues in patients with peripheral neuropathy could lead to plantar foot ulcers. ${ }^{2}$ Up to 600 per 100,000 patients with diabetes develop plantar ulcers leading to amputation, about 10 times the rate in the general poulation. ${ }^{3}$ Increased peak plantar pressure is a predisposing factor for patients to develop plantar ulcers. ${ }^{4}$ 
For those affected by leprosy, protection of anesthetic limbs and prevention of ulcers on the plantar surface has always been given high priority. Unlike non-neuropathic ulcers, which can occur in any part of the body, neuropathic ulcers occur in areas where there is sensory deficit. ${ }^{5}$ Sensory deficit over the pressure prone areas on the plantar surface of the foot lead to pressure ulcers. In low resource leprosy endemic countries, managing plantar ulcers has been a challenge. The cost involved in managing and healing the ulcers is huge and becomes a burden for the families and even for the health care institutions. Loss of daily wages, time away from daily work and even finding appropriate transport to visit a health care centre add to the challenges faced by leprosy affected patients with poor economic resources. A study on leprosy ulcers in Southern India revealed that the unit cost of managing complicated ulcers for an in-patient was 75 Euros for a 30-day care. ${ }^{6}$

Apart from the self-care strategies that have been effectively used in reducing the complications of ulcers, offloading devices have helped heal ulcers by providing sufficient rest to ulcerated area. Appropriate offloading modalities like below knee Total Contact Cast (TCC) and Moulded Double Rocker Shoes (MDRS) are vital for leprosy control programs to offload and heal ulcers. $^{7-9}$

One of the recent scientific advancements is the use of 3-dimensional modeling for fabrication of insoles. The 3-dimensional fabrication of insoles was found to be effective in offloading the foot, although initial investments are required for acquiring data. ${ }^{10,11}$ There is evidence that repeated or prolonged use of the traditional offloading devices like the TCC and MDRS often lead to undesirable social and medical consequences and are sometimes not preferred even by health workers. ${ }^{12,13}$ The other disadvantage with the existing offloading devices is that they are heavy and cumbersome to wear. Even with these disadvantages, these devices are currently used to prevent peak plantar pressures, plantar ulcers and to avoid amputations. ${ }^{14-16}$ Some studies have shown that Total Contact Cast (TCC) is regarded as the gold standard for offloading. ${ }^{17-19}$ One of the conventional and commonly used method of offloading ulcers is using TCC with Bohler iron. ${ }^{20,21}$ The TCC with Bohler iron significantly transfers the weight of the foot and provides rest to the ulcer site, thereby allowing the patient to carry out their activities of daily living. ${ }^{22}$ The TCC with Bohler iron provides axial offloading, where the force passes through the centre of the section and perpendicular to the plane of the section. ${ }^{23}$ This offloading technique aids in healing by reducing the elevated plantar pressure and redistributing forces. ${ }^{24}$ The pressure could thus be offloaded from the foot and redistributed to the shin or the upper part of the leg.

Though studies have established that patients using TCC with Bohler iron can carry out their daily activities, there are certain limitations especially for people who live in villages and do not live under 'perfect conditions'. TCC with Bohler iron is also refused by patients because of its heaviness and its appearance. Designing and developing a light weight, aesthetically appealing, customized offloading devices could help in improving the acceptance of the offloading device and aid in healing of ulcers. It could also help improve acceptability, reduce stigma and can help individuals suffering from ulcers to continue performing their daily activities. This study intends to design a cost effective, light weight offloading device that could improve acceptability and functionality without compromising on the healing of plantar ulcers.

\section{Methods}

The study was carried out at a tertiary leprosy institute where TCC with Bohler iron, MDRS and other such offloading devices are used regularly. Before designing a lighter, low cost and 


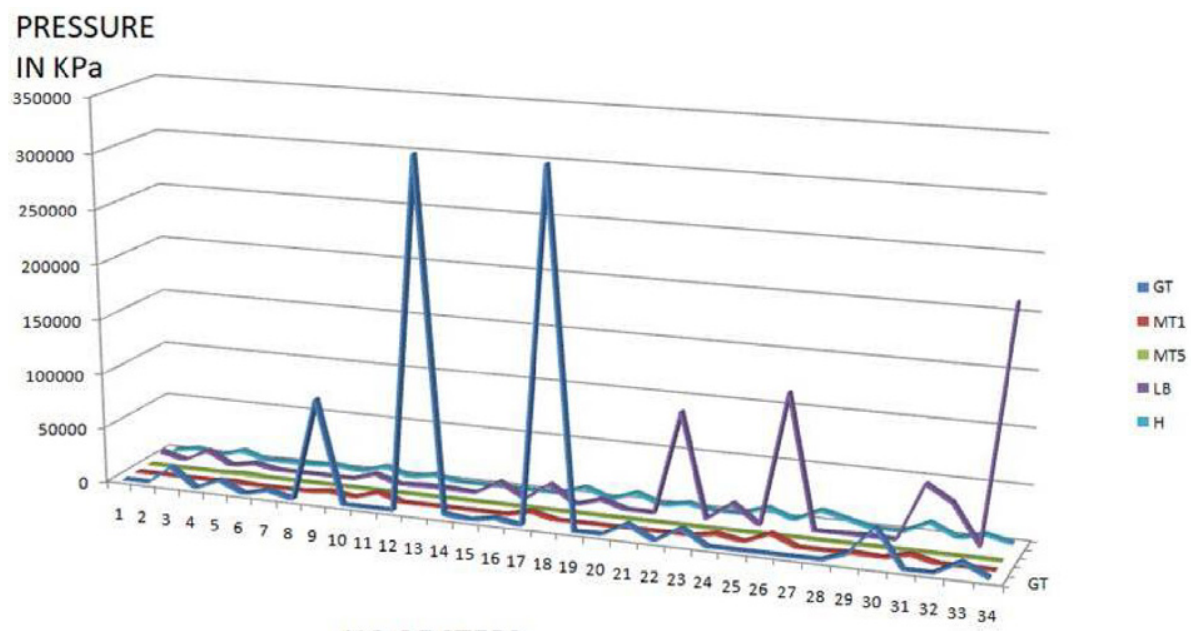

\section{NO OF STEPS}

Figure 1. Shows the pressure distribution of the foot in a traditional Bohler iron.

an aesthetically appealing offloading device, TCC with Bohler iron was tested for its efficacy and strength.

The design specifications of the conventional TCC with Bohler iron were acquired manually using measurement tools to know its length, width and the strength of the materials commonly used. The acquired measurements were then transferred digitally to develop a 3dimensional virtual model of the Bohler iron using Solidworks 2018 software. Solidworks software is commonly used in designing orthotic and prosthetic components and in testing the strength and efficacy of the components to various stresses. ${ }^{25}$ The model of TCC with Bohler iron was simulated and tested using the Solidworks software to enumerate its mechanical behavior.

The Bohler iron was analyzed for its offloading capabilities in real time by measuring the discrete pressures exerted on the pressure prone areas of the foot. The offloading property of the Bohler iron was tested by fixing discrete tactile sensors between the foot and TCC with the Bohler iron.

The pressure data were acquired from the sensors in real time while the patients were walking. ${ }^{26}$ The pressure data from the tactile sensors were used to simulate and test the offloading property of the virtually developed 3-dimensional model. The pressure redistribution identified between the foot and the TCC while using the Bohler iron is graphically represented in Figure 1. The analysis showed that the Bohler iron is effective in offloading the foot at the MT1-1st Metatarsal head, MT5-5th Metatarsal head and the H-Heel. The peak pressures were present in the GT-Great Toe and LB-Lateral Border in spite of using the TCC with Bohler iron. The data acquired through the discrete plantar pressure analysis were used in simulating offloading capabilities of all the 3-dimensional models that were designed and simulated. It also helped to identify ideal offloading device designs.

\section{Results}

The design process was initiated by identifying 2-dimensional (2D) sketches that were compared with the existing offloading models available. The sketches were then converted 
(a)

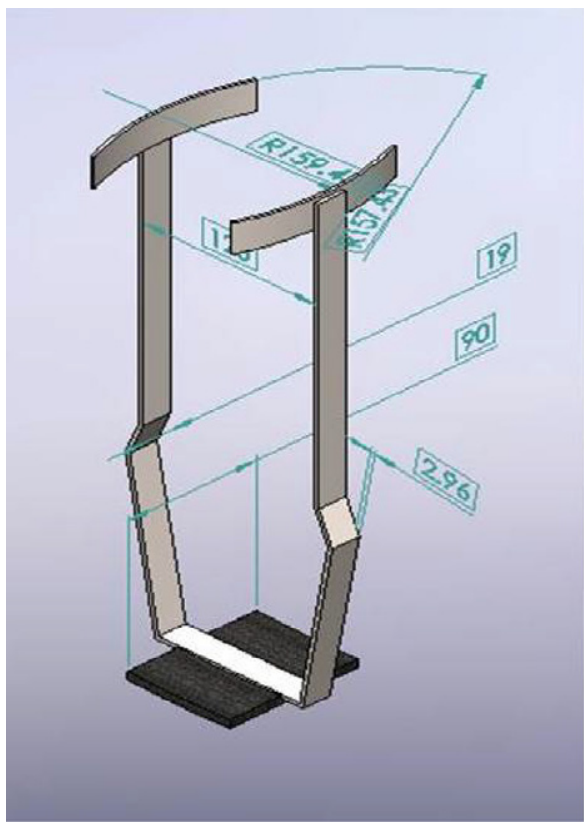

(b)

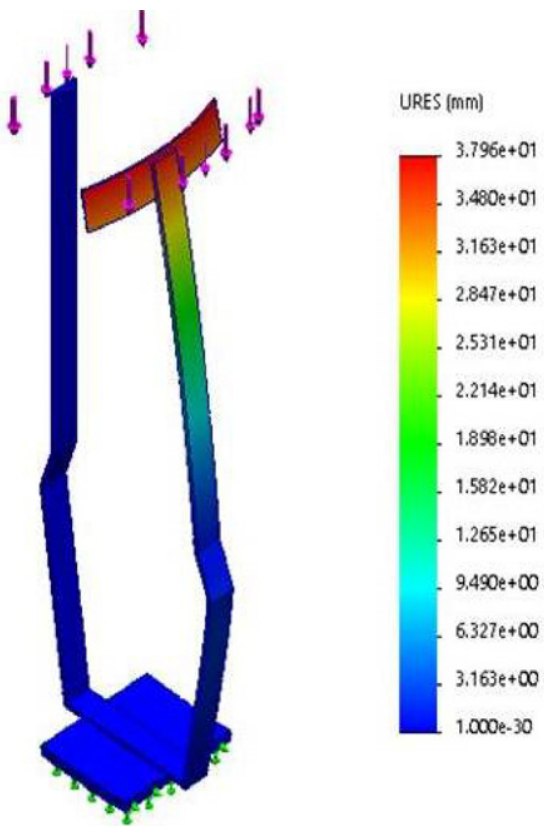

Figure 2. (a) Shows the 3-dimensional model of Bohler iron designed virtually. (b) Shows the simulation results of the Bohler iron tested virtually.

into 3D models by the authors and offloading models were developed. Eight offloading models were then simulated and tested virtually, mimicking the real time scenarios through computer designing and simulation software. ANSYS 2019 R1 was used for simulating and testing the designs developed. ANSYS helped in the analysis of the designed model by setting up appropriate parameters and definitions. The pressure variations and distribution exhibited by the models helped the authors identify appropriate offloading devices.

Figures 2(a) and (b) show the virtual design and the simulation of the conventional Bohler iron.

Apart from the simulation studies, the limitations of the existing conventional offloading devices in carrying out the activities of daily living, the excess weight, difficulties in donning and doffing were considered while designing the new offloading device. Each of the developed designs were then tested for efficacy in offloading. The variables such as the user's body weight, gait pattern and the pressure relieving capabilities were analyzed. Based on the initial analysis, two of the designs with the best outcomes were identified for further studies and prototype development. The designs were identified and approved by the authors, based on their efficacy to offload peak plantar pressure of the foot model in simulation assessments. Parameters such as stress, strain and breaking point of the components were examined. Criteria for selecting the two best designs from the eight initial designs included the use of the minimal number of components that are needed to assemble the offloading device and the low weight of the offloading device that would be fabricated. The details of the comparisons of the different models that were analyzed are provided in Table 1. 
Table 1. Shows the comparison of the analyzed Offloading devices

\begin{tabular}{lccccccccc}
\hline Determinants & \multicolumn{7}{c}{ Model that were simulated } \\
\cline { 2 - 8 } & I $^{*}$ & II $^{*}$ & III & IV & V & VI & VII & VIII \\
\hline $\begin{array}{l}\text { Efficacy to offload the foot } \\
\text { Number of components needed for assembling } \\
\text { the offloading device }\end{array}$ & Yes & Yes & No & Yes & Yes & No & Yes & Yes \\
$\begin{array}{l}\text { Probable weight of the offloading device in } \\
\text { (kilograms), when prototype is fabricated }\end{array}$ & 2.89 & 2.75 & 3.21 & 7 & 3.89 & 4.5 & 3.8 & 6.7 \\
\hline
\end{tabular}

${ }^{*}$ Models selected for fabrication.

\section{DESIGN I}

This design (Figure 3) was developed for effective offloading of the anesthetic feet and to maintain a near normal gait in those using it. The design comprises of a central frame with a foot-piece, calf shell and a foot rest. The calf shell was designed in such a way that it provided adequate freedom of movement and uniform pressure distribution over its surface when a user walks. The cushioned shell is a vital component of the offloading device as it supports the limb. The self-dampening shell in this design helps to maintain the clearance between the leg and the foot rest while using the device. The use of a carbon fiber shell instead of Plaster of Paris to support the calf muscles of the leg provides comfort to the user and also reduces the weight of the device. Donning and doffing of the device is made easy with the removable anterior and posterior parts of the shell. It could even be worn while walking and removed while sleeping, adding to the comfort of the patient, thus improving acceptability and compliance.

This design has a curvature provided to the 'foot-piece' to enforce swift transfer of body weight and stability while walking. The frame and the material it is made from are major factors contributing to the offloading, as it absorbs the ground reaction forces during each step. Although polypropylene also meets all the clinical requirements with its hardness, rigidity and high tensile strength, ${ }^{27}$ carbon fiber was used because of certain unique characteristics, including better durability, light weight and ease of fabrication. ${ }^{28}$ Studies have justified the use of carbon fiber, which provides strength comparable to mild steel. ${ }^{29}$ The effectiveness of carbon fiber in avoiding stress shielding makes it an ideal material for fabricating prostheses. ${ }^{30}$

\section{DESIGN II}

This simple design (Figure 4) was developed to provide complete offloading of the anesthetic ulcerated feet of the patient. The design comprises of a central frame with a foot-piece and a customizable polypropylene calf shell.

This design has angulations at the distal and the proximal parts of the 'foot-piece' of the off loading device. The angulations of the foot-piece would provide ideal weight transfer during gait. Anterior and posterior shells would be custom designed for the calf muscle, to provide rigidity and prevent the slippage of leg from the brace. Polypropylene was used for the anterior and posterior shells as it could be custom molded. Polypropylene (PP) is ideal because of its durability, impact resistance and stiffness. ${ }^{31}$ With a suitable additive, an orthopedic grade PP becomes more flexible and durable under loading conditions. Evidence suggests that PP is an ideal choice for reducing peak pressures in custom molded orthoses. ${ }^{32}$ The foot-piece of the assembly is designed to achieve near normal gait pattern. The curvature at the bottom gives the stability to stand and walk. 


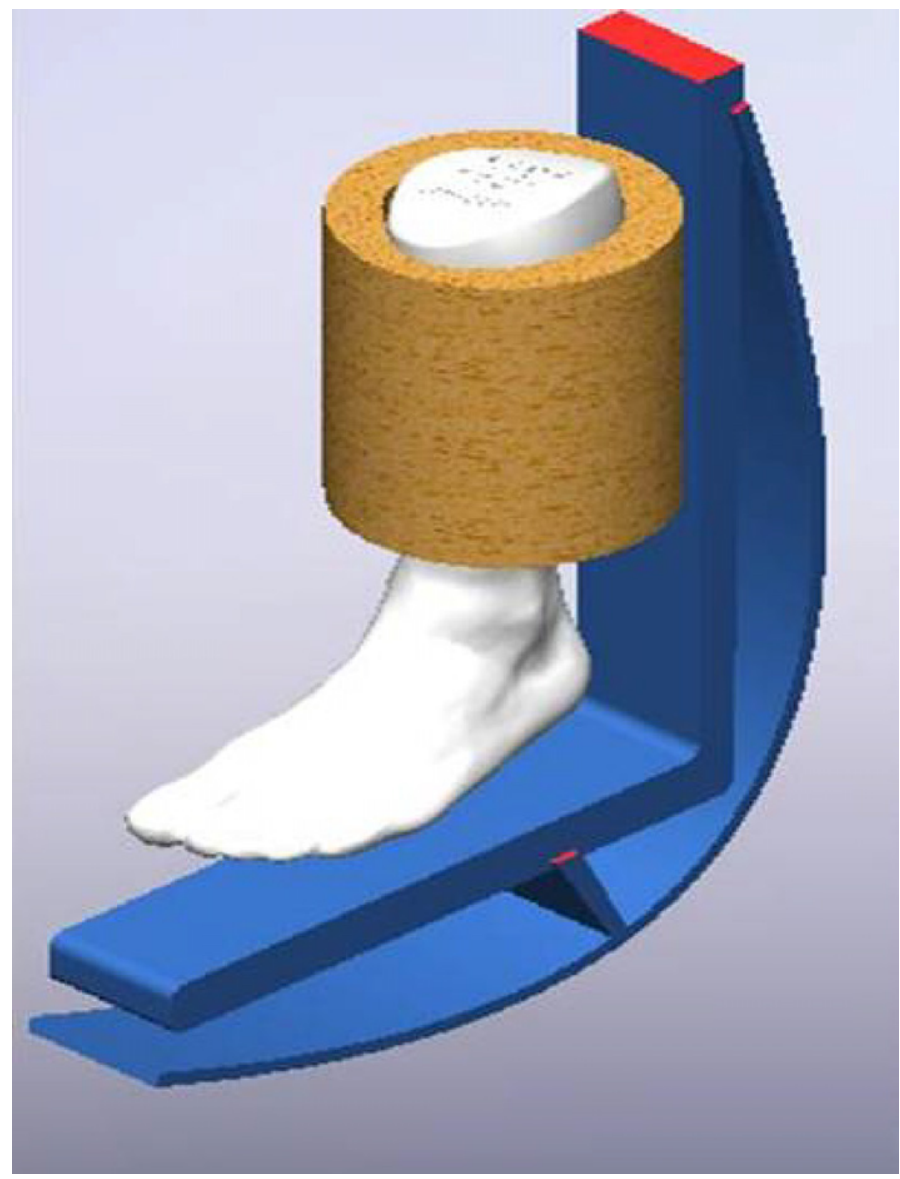

Figure 3. Design I of the offloading device.

\section{Discussion}

Preventing first ulcers and healing of nonhealing chronic plantar ulcers is one of the priorities of every leprosy control program. Offloading ulcers using appropriate devices facilitates the healing process. It is a challenge for health staff to inspect and apply dressings for mid-foot and hind-foot ulcers when TCC with Bohler iron is used. ${ }^{34}$ The computer simulated and designed offloading devices that have been developed could be an ideal replacement for the conventional TCC with Bohler iron. These new designs allow the required mobility and movement of the foot even when the offloading devices are worn. They are also easily removable which is ideal for inspecting and doing daily dressings for the plantar ulcers.

Studies have established that experienced and skilled health workers are needed to apply the TCC cast. ${ }^{34}$ However with the newly developed offloading device the user would be able to easily don and doff them with minimal help. Since the fabricated offloading devices are energy efficient and light weight, it would improve compliance.

In a study carried out by Caravaggi et al., the association between prolonged use of the TCC to muscle atrophy and reduced bone density has been noted. ${ }^{35}$ With the newly designed 


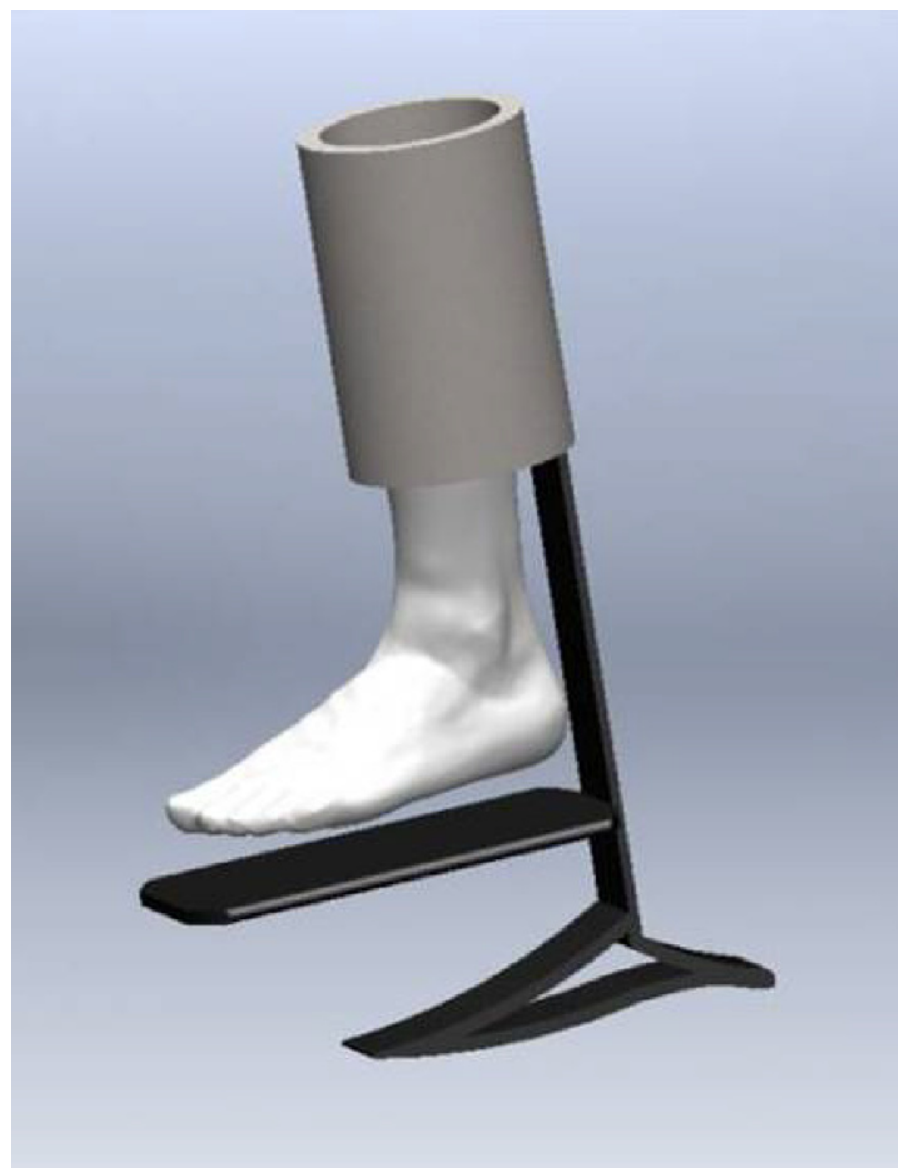

Figure 4. Design II of the offloading device.

offloading device, there is a possibility to remove and reapply by the user themselves and it would help the patients to maintain the muscle tone even while using cast.

Materials for each component of the offloading device were designed and tested through simulation, making them ideal for patients with anesthetic feet. The designs could be bulk manufactured using 3-dimensional printing technology. Orthoses manufactured in bulk could be provided to patients in the field with minor modifications and customization. This rapid prototyping technology is ideal to modify and customize the design for patients with associated deformities of the foot.

\section{Conclusion}

New devices designed and developed for offloading the ulcerated and anesthetic foot could be an ideal replacement for the conventional offloading devices like the TCC with Bohler iron. We recommend further simulation studies of these designs to understand their mechanical behavior and critical failure points. Manufacture of prototypes of these computer simulated designs is underway. Once the fabrication of these prototypes is completed, they will be tested 
on suitable patients. The long term effect on offloading the foot will help in establishing the efficacy of the designs.

\section{Declarations}

\section{Ethics approval and consent to participate}

Ethics review board review and approval was required for this study. All study procedures were approved by the Schieffelin Institute of Health-Research and Leprosy Centre, ethics review board, approval number: 14:05:04.2.

\section{Conflict of interest (COI) statement}

The authors report no conflict of interest.

\section{Funding}

The authors would like to acknowledge the Leprosy Research Initiative (https://www.leprosy research.org) for funding this project through the grant LRI.706.18.29/LRI.

\section{Authors contributions}

Mr. David Prakash Kumar (Corresponding author) contributes on Funding acquisition, Conceptualization, Data curation, Investigation, Supervision and validation, Project Administration and editing.

Dr. Sathish Kumar Paul (Co-author 1) contributes on Funding acquisition, Conceptualization, Data curation, Investigation, Supervision and validation and Project Administration.

Mr. B. Siva (Co-author 2) contributes on Formal analysis, Methodology, Software, Visualization and writing draft.

\section{Patient consent statement}

No patient consent was required as the designs were computer simulated.

\section{Acknowledgements}

The authors acknowledge Dr. Jerry Joshua, Director, SIHRLC Karigiri for his valuable inputs and suggestions.

\section{References}

1 WHO. Weekly epidemiological record - 4 September 2020, 95th Year, No 36, 2020, 95, 417-440.

2 Fernando ME, Crowther RG, Lazzarini PA et al. Plantar pressures are elevated in people with longstanding diabetes-related foot ulcers during follow-up. PLoS One, 2017; 12(8): e0181916. Published 31 August 2017. doi:10.1371/journal.pone.0181916.

3 Moxey PW, Gogalniceanu P, Hinchliffe RJ et al. Lower extremity amputations - a review of global variability in incidence. Diabet Med, 2011; 28: 1144-1153, doi:10.1111/j.1464-5491.2011.03279.x.

4 Armstrong DG, Peters EJ, Athanasiou KA, Lavery LA. Is there a critical level of plantar foot pressure to identify patients at risk for neuropathic foot ulceration? J Foot Ankle Surg, 1998; 37(4): 303-307, doi:10.1016/s 1067-2 516(98)80066-5. PMID: 9710782.

5 Srinivasan H. Management of ulcers in Neurologically impaired feet in leprosy affected persons. In: Schwarz R, Brandsma W (eds), Surgical Reconstruction and Rehabilitation in Leprosy and Other Neuropathies. Kathmandu: Ekta Books Distributors, 2004.

6 Govindarajulu S et al. Operational cost for management of leprosy-related complicated ulcer in charitable hospitals. Lepr Rev, 2015; 86: 283-287. 
7 Pring DJ, Casiebanca N. Simple plantar ulcers treated by below-knee plaster and moulded double-rocker plaster shoe - a comparative study. Lepr Rev, 1982; 53(4): 261-264, doi:10.5935/0305-7518.19820032. PMID: 71548 10 .

8 Diamond JE, Sinacore DR, Muller MJ. Moulded double rocker plaster shoe for treating a diabetic Plantar Ulcer - a case report. Phys Ther, 1987; 67(10): 1550-1552.

9 Joseph B, Joshua S, Fritschii EP. Moulded double rocker plaster shoe for healing a diabetic plantar ulcer. Lepr Rev, 1983; 54(1): 39-44.

10 Paul S, Vijayakumar R, Mathew L, Sivarasu S. Finite element model-based evaluation of tissue stress variations to fabricate corrective orthosis in feet with neutral subtalar joint. Prosthet Orthot Int, 2017; 41(2): 157-163, doi:10.1177/0309364616631344. Epub 2016 Jul 9. PMID: 26979816.

11 Sathish KP, Rekha V, Sudesh S. Customized Insole fabrication for foot deformities in Leprosy patients. $J$ Med Dev, 2014; 8(2): 020950. doi:10.1115/1.4027065.

12 Cross $\mathrm{H}$ et al. The efficacy of podiatric orthoses as an adjunct to the treatment of plantar ulceration in leprosy. Lepr Rev, 1995; 66(2): 144-157.

13 Wu SC, Jensen JL, Weber AK, Robinson DE, Armstrong DG. Use of pressure offloading devices in diabetic foot ulcers: do we practice what we preach? Diabetes Care, 2008; 31(11): 2118-2119, doi:10.2337/dc08-0771.

14 Raspovic A, Landorf KB, Gazarek J, Stark M. Reduction of peak plantar pressure in people with diabetesrelated peripheral neuropathy: an evaluation of the DH Pressure Relief Shoe ${ }^{\mathrm{TM}}$. J Foot Ankle Res, 2012; 5(1): 25, Published 1 October 2012. doi:10.1186/1757-1146-5-25.

15 Westra M, van Netten JJ, Manning HA, van Baal JG, Bus SA. Effect of different casting design characteristics on offloading the diabetic foot. Gait Posture, 2018; 64: 90-94, doi:10.1016/j.gaitpost.2018.05.022.

16 Van De Weg FB, Van Der Windt DA, Vahl AC. Wound healing: total contact cast vs. custom-made temporary footwear for patients with diabetic foot ulceration. Prosthet Orthot Int, 2008; 32(1): 3-11, doi:10.1080/030936 40701318672 .

17 Armstrong DG, Nguyen HC, Lavery LA, van Schie CH, Boulton AJ, Harkless LB. Off-loading the diabetic foot wound: a randomized clinical trial. Diabetes Care, 2001; 24(6): 1019-1022, doi:10.2337/diacare.24.6.101 9 [published correction appears in Diabetes Care, 2001; 24(8): 1509].

18 American Diabetes Association. Consensus Development Conference on Diabetic Foot Wound Care: 7-8 April 1999, Boston, Massachusetts. American Diabetes Association. Diabetes Care, 1999; 22(8): 1354-1360, doi:10.2337/diacare.22.8.1354.

19 Alexiadou K, Doupis J. Management of diabetic foot ulcers. Diabetes Ther, 2012; 3(1): 4, doi:10.1007/s13300 -012-0004-9. Epub 20 April 2012. PMID: 22529027; PMCID: PMC3508111.

20 Venkatakrishnan S, Zachariah K et al. A description of a modified Bohler iron walking cast in the management of plantar ulcers. J Foot Ankle Surg (Asia-Pacific), 2019; 6(1): 10-12.

21 Paul MSK, Kumar DP, Govindasamy K. Physical rehabilitation in leprosy. In: Scollard DM, Gillis TP (eds), International Textbook of Leprosy. American Leprosy Missions, 26 January 2019, posting date.

22 Berwin JT, Burton TM, Taylor J, McGregor AH, Roche A. Plantar loading forces while walking in a below-knee cast with an attached load bearing frame. Foot Ankle Int, 2015; 36(6): 722-729, doi:10.1177/10711007155722 58.

23 Thakur J, Kumar R, Basu D, Hansda K, Das Munshi B, Sasthi Narayan N, Maity S, Bahrus K, Richa R, Kumar N. Prevalence of diabetic foot syndrome and its determinants among Type 2 Diabetes Mellitus patients attending Integrated Diabetes \& Gestational Diabetes Clinic of a Tertiary Health Care Level Hospital of Eastern India, 2019. doi:10.9790/0853-1801092429.

24 Saikia P, Hariharan R, Shankar N, Gaur AK, Jose NM. Effective and economic offloading of diabetic foot ulcers in india with the Bohler iron plaster cast. Indian J Surg, 2016; 78(2): 105-111, doi:10.1007/s12262-015-13273.

25 Zlata J, Remzo D, Haris D. Chapter 5 - Prosthetic design and prototype development. In: Zlata J, Remzo D, Haris D (eds), Active Above-Knee Prosthesis. Academic Press 2020; pp. 155-199, ISBN 9780128186831, doi:10.1016/B978-0-12-818683-1.00005-6.

26 Sathish Paul M, Siva B. Peak plantar pressure analysis using customized tactile sensory feedback system - A short report. Lepr Rev, 2021; 92(1): 82-87, doi:10.47276/lr.92.1.82.

27 Quintero-Quiroz C, Pérez VZ. Materials for lower limb prosthetic and orthotic interfaces and sockets: Evolution and associated skin problems. Rev Fac Med, 2019; 67: 117-125.

28 Hachisuka K, Makino K, Wada F, Saeki S, Yoshimoto N, Arai M. Clinical application of carbon fibre reinforced plastic leg orthosis for polio survivors and its advantages and disadvantages. Prosthet Orthot Int, 2006; 30(2): 129-135, doi:10.1080/03093640600574474.

29 Sun W, Luo Y, Sun H. Experimental studies on the elastic properties of carbon fiber reinforced polymer composites prefabricated of unidirectional carbon fiber fabrics and a modified rule of mixtures in the parallel direction. Adv Compos Lett, 2018; 27: 34-43, doi:10.1177/096369351802700104. 
30 Koumoulos EP, Trompeta A-F, Santos R-M, Martins M, Santos C, Iglesias V, Böhm R, Gong G, Chiminelli A, Verpoest I, Kiekens P, Charitidis CA. Research and development in carbon fibers and advanced highperformance composites supply chain in Europe: a roadmap for challenges and the industrial uptake. J Compos Sci, 2019; 3: 86, doi:10.3390/jcs3030086.

31 Maddah, HA. Polypropylene as a promising plastic: a review. Am J Polym Sci, 2016; 6(1): 1-11, doi:10.5923/ j.ajps.20160601.01. ISSN: 2163-1344

32 David CS, Martha LS. Sheet plastics and their applications in orthotics and prosthetics. Orthotics Prosthetics, 1984; 38(4): 41-48.

33 Venkatakrishnan S, Zachariah $\mathrm{K}$ et al. A description of a modified bohler iron walking cast in the management of plantar ulcers. J Foot Ankle Surg (Asia-Pacific), 2019; 6(1): 10-12.

34 Rogers LC. Overcoming barriers to adopting the total contact cast in your practice. Podiatr Today, 2015; 28(8): p.n.p. [Google Scholar].

35 Caravaggi C, Faglia E, De Giglio R et al. Effectiveness and safety of a nonremovable fiberglass off-bearing cast versus a therapeutic shoe in the treatment of neuropathic foot ulcers: a randomized study. Diabetes Care, 2000; 23(12): 1746-1751. 